\title{
Nanostructured System: A Novel Approach for Pharmacotherapy of Pulmonary Diseases
}

\section{Jagadevappa Patil}

VT's Shivajirao S Jondhle College of Pharmacy, Asangaon-412 601, Thane, Maharashtra, India

*Corresponding author: Jagadevappa Patil, VT's Shivajirao S Jondhle College of Pharmacy, Asangaon-412 601, Thane, Maharashtra, India, Tel: 9448816812 ; E-mail: pharmajspatil@gmail.com

Received date: September 04, 2015; Accepted date: September 07, 2015; Published date: September 11, 2015

Copyright: @ 2015 Patil J. This is an open-access article distributed under the terms of the Creative Commons Attribution License, which permits unrestricted use, distribution, and reproduction in any medium, provided the original author and source are credited.

\section{Editorial}

\section{Introduction}

Nanotechnology is the art and technique provides new materials on atomic or molecular scales with many potential applications in the field of drug development, clinical medicine and biomedical research. The novelty and uniqueness of nanostructured materials majorly depends on size-related properties which offer the possibility to develop both new therapeutic and diagnostic tools. In the last few years, problems of health care sectors are addressing through development of nanostructured medicines. Hence, applied nanotechnology has gained more attention to solve the medical problems through development of nanomedicine. A novel paradigm in pharmacotherapy has been displayed due to unique characteristic of nanostructured materials. Cell targeted drug delivery is an amazing approach could be achieved through design of nanosystems which have an ability to hold the drugs in their core. Nanostructured materials coated with polymers, proteins like albumin and solid-lipid particles have been used as carriers. However, the drug entities can also be coupled with these organ and/or cell specific carriers either trapped within or deposited in subsurface oil layers intended to deliver the drugs to the specific organs, tumors and cells. The strategies of delivering nanostructured targeting carriers can be utilized to concentrate drugs in a targeted site or selected organs/cells/tissues thus reducing systemic side effects and toxicity. In extension to these unique therapeutic options, nanoparticle-based systems can also be effectively used as diagnostic imaging tools.

Pulmonary disorders are increasing pathologic entities in the ageing population. The modern changing lifestyle of public expects better quality of life with advanced and affordable health care. There is no synchronized improvement in our understanding of the functioning of the human body at the molecular/nano level and our diagnostic and therapeutic options for the effective treatment of severe and chronic diseases. Pulmonary diseases are serious challenges to be dealt with. To speed up the novel diagnostic and therapeutic remedies multidisciplinary approaches that bring together material and medical scientists is need of the hour [1]. Pulmonary route is most commonly used and well accepted for non-invasive drug delivery for many lung diseases and has become an alternative target and of tremendous scientific and biomedical interest in the health care research area. Lung has the capability to absorb active pharmaceutical compounds delivered either for systemic or local treatment. The pharmaceutical scientists are taking advantage of large peripheral surface area (100 $\mathrm{m}^{2}$ ) of lung for absorption and thinner (0.1-0.2 $\left.\mu \mathrm{m}\right)$ alveolar epithelium providing shorter distance of air-blood exchange passage [2]. Specially designed drug delivery systems containing inhaled drugs can be efficiently delivered to the respiratory tract to achieve sustained release pattern. The advantages for sustained release drug delivery to the respiratory tract are numerous, and include extended duration of action, reduction in drug use, improved management of therapy, improved compliance, reduction in side effects together with potential cost savings that exist for sustained release therapy [3]. In this regard, here an attempt was made to partially describe the applications of applied nanotechnology to medical problems through nanostructured medicine for pulmonary diseases.

\section{Pulmonary Disorders}

Nanostructured medicines designed via applied nanotechnology provide new concepts for diagnoses and therapies. These new concepts are interrelated between three themes, molecular imaging, targeted drug delivery including controlled release, and regenerative medicine. The ability of a nanostructured system to easily administer through airways make it possible to treat number of respiratory diseases such as obstructive lung diseases, genetic disorders affecting the airways, infectious diseases including tuberculosis, and cancer.

\section{Nanostructures Used in Obstructive Lung Diseases}

The major cause of disability and death are mainly due to the obstructive airway diseases, chronic obstructive pulmonary disease and bronchial asthma. Since the main solution of drug delivery in the form of nano-carrier system for the treatment of such diseases is the pulmonary route. Experimental study has already been reported regarding use of nanostructures in the treatment of asthma [4]. In contrast to the above reported study focusing on the use of nanostructures in bronchial asthma, no much comprehensive reports are available on potential use of nanostructures for the treatment of chronic obstructive pulmonary diseases except for new compounds are developed [5].

\section{Nanostructures Used in Airways Affecting Genetic Disorders}

Cystic fibrosis, a kind of airways affecting disorder can also be treated successfully using nanostructured materials. The mucus hyper secretion and deficient channel protein which are responsible for cystic fibrosis may be targeted by nanostructures; cystic fibrosis seems to be an ideal candidate for the therapeutic use of such systems. In a one of the study, a gelatine and DNA nanoparticle coacervate containing chloroquine and calcium has been developed as a gene delivery vehicle. In this vehicle the cell ligand transferring is covalently bound to gelatine [6]. Therefore, nanostructures might be an attractive candidate to deliver new therapeutic compounds in cystic fibrosis. 


\section{Nanostructures Used in Infectious Diseases}

Many chronic lung diseases caused by bacterial and viral infections which lead to a progressive destruction of lung tissue and finally respiratory failure [7]. Under such circumferences, achieving high concentration of antimicrobial agents in the lung is the most important which facilitate successful eradication of bacteria. The major local site of lung mainly the alveolar and bronchial epithelium is a site with major opportunity for drug delivery and therapy [8]. Direct delivery of antibiotics to the lower airways by aerosol administration has potential advantages: deposition to the alveolar site of the infection can reach high local concentration [2,9]. The use of nanostructures to deliver such anti-infective drugs is of particular interest and a number of studies have already reported on this issue [10].

\section{Nanostructures Used in Pulmonary Tuberculosis}

Nanostructured medicines have also been evaluated for their potential use in antimicrobial therapy. One of the first reported studies on the issue of nanoparticle-encapsulated antitubercular drugs as potential oral drug delivery systems against murine tuberculosis [11]. One of the major drawbacks with longer duration tuberculosis treatment is patient non-compliance. A reduction in the frequency of dosing using nanostructured medicine might therefore lead to a significant improvement in the therapy. Overall these studies demonstrated that the pulmonary administration of drug encapsulated respirable nanostructures can be used experimentally to improve pharmacological management of pulmonary tuberculosis.

\section{Nanostructures Used in Cancer Therapy}

In last few years treatment of lung cancer by cancer gene therapy has been demonstrated to have beneficial effects in experimental and in preclinical trials. Due to the host-immunity response against the gene delivery vector and the transgene made limited applications of this approach in the treatment of localizes lung cancer. In this context, studies are currently focusing on the development of novel, nonimmunogenic gene delivery vectors [12].

\section{Conclusion}

Our tremendous advanced approach in understanding of the human anatomy and physiology at the molecular level is little not synchronized with the alternative options of diagnostic and therapeutic remedies for the effective treatment of severe and chronic diseases. Although, we are far ahead in development of novel drug delivery devices, various pulmonary disorders are still presenting serious challenges which are to be dealt with. It is very much essential to bring material and pharmaceutical scientists together to find new avenues through multidisciplinary approaches which can speed up the development of new diagnostic and therapeutic solutions. Nanotechnology based on material science has provided new drug delivery systems which can deliver the variety of drugs to particular organ or tissues or cells including the respiratory tract. Better pharmacotherapy of pulmonary diseases can be achieved through rightful utilization of concepts of nanomedicine. The nanostructured system can be effectively used to encapsulate large number of bioactive agents and therefore, nearly every pulmonary disease may be targeted using this new field of research. Moreover, future clinical studies are required to bridge the huge gap between concept of nanostructures and clinical reality. In order to address health care problems, it needs to develop the precise and effective diagnostic and therapeutic potential of nanostructured medicine.

\section{References}

1. Pison U, Welte T, Michael G, David AG (2006) Nano medicine for respiratory diseases. Eur J Pharmacol 533: 342-350.

2. Patil JS, Devi VK, Devi K, Sarasija S (2015) Formulation and Evaluation of Novel Spray-dried Alginate Microspheres as Pulmonary Delivery Systems of Rifampicin in Rats. Indian J Pharm Edu Res 49: 320-328.

3. Patil JS, Devi VK, Devi K, Sarasija S (2015) A novel approach for lung delivery of rifampicin-loaded liposomes in dry powder form for the treatment of tuberculosis. Lung India 32: 331-338.

4. John AE, Lukacs NW, Berlin AA, Palecanda A, Bargatze RF, et al. (2003) Discovery of a potent nanoparticle P-selectin antagonist with antiinflammatory effects in allergic airway disease. FASEB J 17: 2296-2298.

5. Chung KF, Caramori G, Groneberg DA (2004) Airway obstruction in chronic obstructive pulmonary disease. N Engl J Med 351:1459-1461.

6. Truong-Le VL, Walsh SM, Schweibert E, Mao HQ, Guggino WB, et al. (1999) Gene transfer by DNA-gelatin nanospheres. Arch Biochem Biophys 361: 47-56.

7. Koch C, Hoiby N (1993) Pathogenesis of cystic fibrosis. Lancet 341: 10651069.

8. Ramsey BW (1996) Management of pulmonary disease in patients with cystic fibrosis. N Engl J Med 335: 179-188.

9. Honeybourne D (1994) Antibiotic penetration into lung tissues. Thorax 49: 104-106.

10. Groneberg DA, Peiser C, Dinh QT, Matthias J, Eynott PR, et al. (2003) Distribution of respiratory mucin proteins in human nasal mucosa. Laryngoscope 113: 520-524.

11. Pandey R, Sharma A, Zahoor A, Sharma S, Khuller GK, et al. (2003) Poly (DL -lactide-co-glycolide) nanoparticle-based inhalable sustained drug delivery system for experimental tuberculosis. J. Antimicrob. Chemother 52: 981-986.

12. Gopalan B, Ito I, Branch CD, Stephens C, Roth JA, et al. (2004) Nanoparticle based systemic gene therapy for lung cancer: molecular mechanisms and strategies to suppress nanoparticle-mediated inflammatory response. Technol Cancer Res Treat 3: 647-657. 